\title{
Primary hepatic leiomyoma in a Chinese female patient without underlying disease: a case report
}

\author{
Baoxing Jia ${ }^{1}$, Zhe Jin ${ }^{1}$, Pin Gao ${ }^{2}$ and Yahui Liu ${ }^{1 *}$
}

\begin{abstract}
Background: Primary hepatic leiomyoma $(\mathrm{PHL})$ is a rare manifestation of tumors in the liver; it is mainly characterized by its origin in the mesenchymal tissue. To date, the mechanisms underlying the pathogenesis of this disease remain unclear, however most reported PHL patients suffer from acquired immunity deficiency syndrome (AIDS) or take immunosuppressive medications after organ transplantation.

Case presentation: In this case report we describe a rare case of PHL in a middle-aged Chinese woman who was asymptomatic with no history of hepatitis or other liver disease. She had no history of immune suppression medication therapy. In view of the benign features of the hepatic lesion, along with our implementation of the respecting the patience choices, a laparoscopic partial hepatectomy of the right lower liver was performed, which appeared to be highly effective and give a good prognosis.

Conclusions: Clinical characteristics of the patient should be compared to previously reported aspects of this disease to reach a clear diagnosis. Moreover, although PHL is extremely rare, it should still be considered a possibility. Surgical intervention is effective in treating this disease.
\end{abstract}

Keywords: Leiomyoma, Liver neoplasm, Primary hepatic leiomyoma, Laparoscopic hepatectomy

\section{Background}

Primary hepatic leiomyoma (PHL), a rare manifestation of tumors in the liver [1, 2]; is mainly characterized by its mesenchymal tissue origin in the liver, and no cases of leiomyoma are identified in the gastrointestinal and urinary tracts or elsewhere in the body [3].

To date, the pathogenic mechanisms underlying this disease remain unclear. While it has been postulated that the neoplasia may arise from atypical growth of hepatic vessels and abnormal proliferation of bile ducts $[1,4,5]$ this has not been well validated by scientific research.

Since 1926, when the first case of PHL was described, most PHL cases have been reported among patients suffering from acquired immunity deficiency syndrome (AIDS) or AIDS in combination with Epstein-Barr virus (EBV) infection, or taking immunosuppressive medications

\footnotetext{
*Correspondence: liuyahuisci@163.com

1 Department of Hepatobiliary and Pancreatic Surgery, the First Hospital of

Jilin University, Changchun 130021, Jilin, China

Full list of author information is available at the end of the article
}

after organ transplantation [2, 6]. To our knowledge, PHL in immunocompetent patients is extremely rare.

In this case report, we describe an unusual case of PHL in a middle-aged Chinese woman who was asymptomatic with no immunosuppressive disorders, no history of hepatitis or other liver disease, and no history of immunosuppressive medication use. After a successful laparoscopic partial hepatectomy, the patient has an excellent prognosis.

\section{Case presentation \\ Patient description}

A 46-year-old Chinese woman consulted a physician in our department for an intrahepatic mass incidentally detected by an abdominal ultrasound (US) during her annual physical examination. After a 7-cm hypoechoic mass in the right lobe of the liver was identified and confirmed, the patient was admitted to our hospital for further investigation and treatment of the hepatic lesion. She reported no immunosuppressive medication use and no history of liver disease or surgery. 

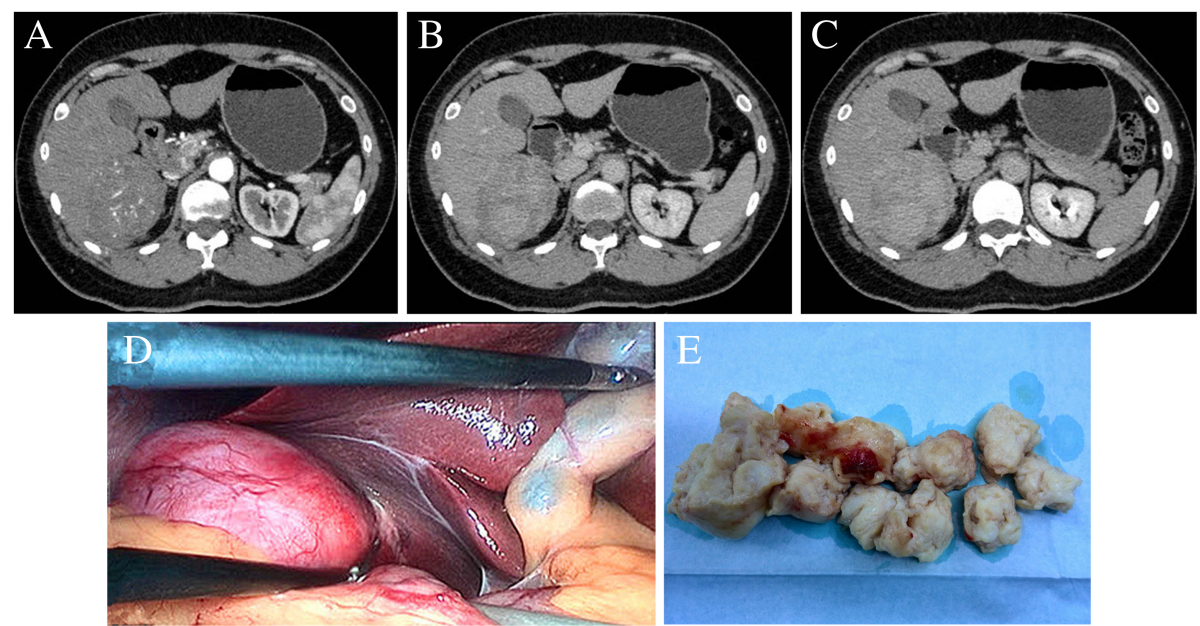

Fig. 1 Abdominal CT scan images of the patients. CT images demonstrate (a) heterogenous enhancement in the artery phases; $\mathbf{b}$ progressive enhancement in the portal venous phases; and $\mathbf{c}$ prolonged enhancement in the balanced phases. $\mathbf{d}$ An image during the laparoscopic partial hepatectomy. e Tumor tissue samples

\section{Clinical examination}

No mass and discomfort and no signs of other clinical symptoms were detected during the abdominal examination. Hematological and serum biochemical profiles were within normal ranges. Tumor markers including alphafetoprotein (AFP), carcinoembryonic (CEA), carbohydrate antigens 199 (CA199), and carbohydrate antigens 125 (CA125) were also normal.
Further investigation of the hepatic lesion with computed tomography (CT) revealed a slightly hypodense $6.5 \times 7.2 \mathrm{~cm}$ mass in the right lobe of the liver with heterogenous enhancement of arterial phase, and prolonged enhancement through portal venous phase and the lesion (Fig. 1a, b, c). No other abnormalities were identified in the remaining liver tissue and elsewhere the abdomen.
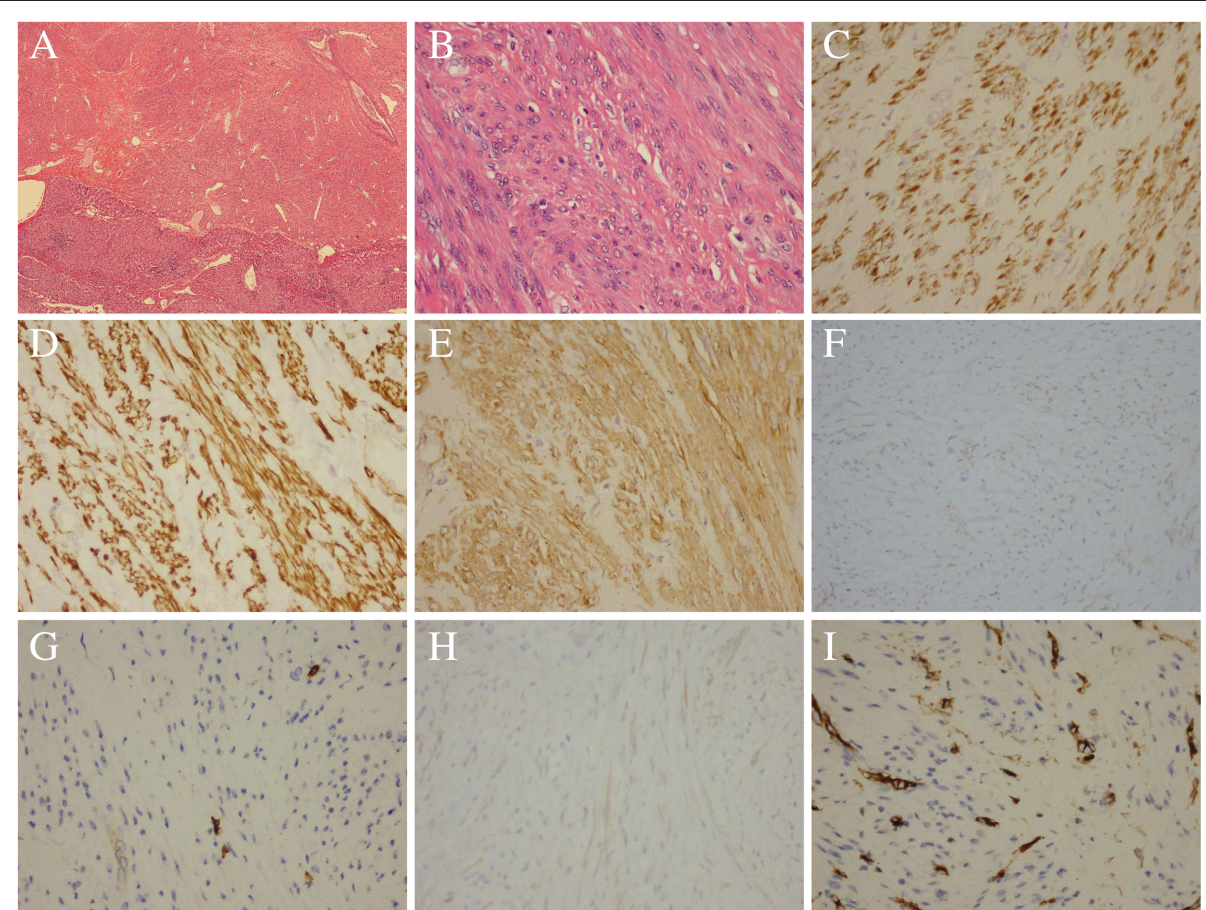

Fig. 2 Characterization of primary hepatic leiomyoma staining. a Tissue sample staining $\times 4$; $\mathbf{b}$ tissue sample staining $\times 40$; $\mathbf{c}$ desmin $(+)$; $\mathbf{d ~ H}$ caldesmon (+); e SMA (+); f Dog-1 (-); g CD117 (-); h; S-100 (-); i CD34 (-) 
As the CT imaging was inconclusive, further evaluation with magnetic resonance imaging (MRI) as well as USguided fine needle aspiration (FNA) biopsy of the tumor for pathological examination was recommended. However, the patient preferred an operation without additional preoperative assessment of the lesion.

\section{Treatment and outcome}

In view of the benign features of the hepatic lesion and in accordance with our patient's choice of treatment, we carried out laparoscopy (Olympus, Tokyo, Japan). A 12-mm optical trocar at the umbilicus as well as three other trocars was used. Two 12-mm trocars were used through the epigastrium and right upper abdomen. A 5-mm trocar was used through the right abdomen near the anterior axillary line. After a laparoscopic exploration and US, a solid $6.5 \mathrm{~cm} \times 7.0 \mathrm{~cm} \times 7.5 \mathrm{~cm}$ mass was clearly located in the segment 6 of the liver and outwardly protruding from the liver surface (Fig. 1d). We decided to perform laparoscopic partial hepatectomy. Hepatic parenchyma was performed using an UltraCision Harmonic Scalpel (Ethicon Endo-Surgery, Cincinnati, OH, USA). The branches of the Glissonian pedicles and hepatic vein within the liver were clipped using a Hem-o-lok ${ }^{\bullet}$ (Teleflex Medical, Morrisville, NC, USA) and a titanium clip (TSCS, Hangzhou, China), and then transected. We put the surgical specimen into a laparoscopic disposable specimen bag (Xueli, Nanchang, China) through the epigastrium incision, and to avoid expanding the incision we cut the specimen into small pieces in the bag before taking them out (Fig. 1e). Finally, the surgical area was carefully examined and a drainage tube was not placed. Seven days after surgery, the patient was discharged from hospital. After a 2-year follow-up, no recurrence or metastasis occurred.

\section{Final diagnosis}

Postoperative pathological examination revealed a benign smooth muscle tumor derived from the mesenchymal tissue of the liver with clearly visible margins and with no evidence of necrosis or tumor invasion (Fig. 2a, b). Immunohistochemistry ( $\mathrm{IH})$ showed strong reactivity for smooth muscle actin (SMA) $(+)$, desmin $(+)$, and $\mathrm{H}$-caldesmon $(+)$ (Fig. 2c, d, e), but not for Dog-1 (-), CD117 (-), S-100 (-), and CD34 (-) (Fig. 2f, g, h, i). According to the diagnostic criteria for PHL, the patient was finally diagnosed with PHL.

Table $1 \mathrm{PHL}$ without immune-compromised cases in medical literature

\begin{tabular}{|c|c|c|c|c|c|c|}
\hline & Author & Year & Age/Sex & Symptoms & Location/Size $(\mathrm{cm})$ & Treatment \\
\hline 1 & Demel [7] & 1926 & $42 / F$ & RUQ pain & $\mathrm{RL} / 12$ & Laparotomy \\
\hline 2 & Rios-Dalenz [8] & 1965 & $87 / F$ & RUQ pain & LLL/NS & Autopsy \\
\hline 3 & Ishak et al. [9] & 1975 & $64 / M$ & Abdominal mass & RL/NS & Laparotomy \\
\hline 4 & Hawkins et al. [3] & 1980 & $66 / M$ & Abdominal mass & $\mathrm{LL} / 13$ & Left hepatectomy \\
\hline 5 & Hollands et al. [5] & 1989 & $17 / M$ & UA pain & $\mathrm{LL} / 9$ & Left hepatectomy \\
\hline 6 & Herzberg et al. [10] & 1990 & $30 / F$ & RUQ pain & $\mathrm{RL} / 19$ & Partial right hepatectomy \\
\hline 7 & Bartoli et al. [11] & 1991 & $34 / F$ & None & $\mathrm{RL} / \mathrm{NS}$ & Right hepatectomy \\
\hline 8 & Reinertson et al. [12] & 1992 & $32 / F$ & RUQ pain & $\mathrm{LL} / 10$ & Left hepatectomy \\
\hline 9 & Yanase et al. [13] & 1999 & $59 / F$ & Liver dysfunction & $\mathrm{RL} / 13$ & Right hepatectomy \\
\hline 10 & Mesenas et al. [14] & 2000 & $59 / \mathrm{M}$ & None & $\mathrm{RL} / 3.6$ & Segmentectomy (S5) \\
\hline 11 & Belli et al. [4] & 2001 & $67 / F$ & Abdominal mass & $\mathrm{RL} / 30$ & Right extended resection \\
\hline 12 & Kanazawa et al. [15] & 2002 & $31 / \mathrm{M}$ & None & $\mathrm{LLL} / 3.5$ & LLL resection \\
\hline 13 & Beuzen et al. [16] & 2004 & $36 / F$ & RUQ pain & $\mathrm{LLL} / 5$ & LLL resection \\
\hline 14 & Imasato et al. [17] & 2005 & $61 / F$ & None & $\mathrm{CL} / 4.5$ & Right hepatectomy \\
\hline 15 & Urizono et al. [18] & 2006 & $71 / \mathrm{M}$ & None & $\mathrm{CL} / 3$ & Partial hepatectomy \\
\hline 16 & Marin et al. [19] & 2008 & $64 / F$ & None & $\mathrm{RL} / 3$ & Right hepatectomy \\
\hline 17 & Sousa et al. [20] & 2009 & $61 / F$ & Dyspepsia & LLL/9.5 & Left hepatectomy \\
\hline 18 & Kalil et al. [21] & 2009 & $44 / F$ & Abdominal mass & $\mathrm{RL} / 7$ & Atypical resection \\
\hline 19 & Santos et al. [22] & 2011 & $28 / F$ & None & $\mathrm{RL} / 5.5$ & Segmentectomy \\
\hline 20 & Perini et al. [23] & 2012 & $45 / F$ & RUQ pain & $\mathrm{RL} / 16.5$ & Segmentectomy \\
\hline 21 & Vyas et al. [24] & 2015 & $20 / F$ & UA pain & LLL/8 & LLLL resection \\
\hline 22 & Navarro et al. [25] & 2015 & $44 / F$ & None & RL/NS & Segmentectomy $(\$ 5,7,8)$ \\
\hline
\end{tabular}

RUQ Right upper quadrant, RL Right lobe, LLL Left lateral lobe, NS Not stated, LL Left lobe; 


\section{Discussion and conclusions}

Since 1926 when the first case of PHL was described, just 22 immunocompetent cases have been reported in the medical literature (Table 1). Here we reported the 23rd case. The average age was 48.17 years and the male to female ratio was 6:17. The mass size ranged from $3 \mathrm{~cm}$ to $30 \mathrm{~cm}$. Thirteen cases were found in the right lobe of liver, eight cases in the left lobe, and two cases in the caudate lobe.

A PHL diagnosis needs to satisfy the following criteria: (1) the tumor originates from the hepatic mesenchymal tissue; and (2) there are no primary tumors elsewhere in the body. However, despite this clear criteria for identifying this rare type of intrahepatic tumor, a successful preoperative diagnosis of PHL is challenging, mainly due to imaging features being similar with other benign hepatic tumors as well as a lack of specific characteristics to guide physical and laboratory examinations [5, 8, 23].

Several common imaging patterns have been identified in PHL patients. On US scans, PHL appears as heterogenous hypoechoic nodules [16, 18, 20]; a finding also seen in our case. In CT imaging, hypodense lesions are widely reported with marked enhancement in the arterial and portal venous phases, occasionally in the peripheral phase, and with prolonged enhancement in the equilibrium phase $[5,16,20]$. On MRI, the PHL usually presents a lower signal in T1-weighted images and a higher signal in the T2-weighted images [2, 18, 23]. In addition, lesion characteristics (irregular margins) on hepatic angiography have been reported in a few PHL cases $[15,18]$. Non-invasive, preoperative imaging patterns on US, CT, MRI, and angiography can not define PHL.

Of the reported PHL cases in the literature, Sousa and colleagues achieved an accurate diagnosis of PHL in a healthy middle-aged woman by undertaking an imagingguided fine needle aspiration (FNA) and a $18 \mathrm{G}$ tru-cut liver biopsy of the tumor tissue [20]. Sadler and colleagues reported two cases with preoperative diagnosis on liver biopsy: one case with mesenchymal mixed tumor of the liver but an accurate diagnosis could be not reached in the other case [26]. In our patient, like most reported cases, a non-invasive, preoperative diagnosis with imaging features was inconclusive.

After a postoperative pathological examination was carried out on the biopsy specimen, the pathological features were noteworthy. A benign smooth muscle tumor containing the mesenchymal tissue with clear margins was visualized. Positive staining for SMA, which is observed in most reported PHL cases and is a hallmark of PHL, was confirmed in this case. Moreover, positive staining for desmin and $\mathrm{H}$-caldesmon, as observed in some reported PHL cases [14], was also noticed in our patient, whereas vimentin was negatively expressed in the tumor tissue (also reported in some
PHL cases). In addition, negative staining for Dog-1 and CD117 distinguished PHL from the gastrointestinal stromal tumors (GIST), while negative reactivity for S-100 ruled out leiomyoma of the central nervous system and CD34 excluded vascular leiomyoma. The patient thus fulfilled the two criteria and was eventually diagnosed with PHL.

In this case, given the benign features of the hepatic lesion, which usually signifies treatment with surgery [27] as well as our respect for the patient's preferred choice of treatment, we performed a laparoscopic partial hepatectomy of the right lower liver [24]. The procedure appeared to be highly effective in this case, and our patient has a good prognosis and is expected to have no recurrence in the long term.

In conclusion, the imaging and pathological features of the patient should be added to previously reported aspects of this disease. Moreover, although PHL is extremely rare, it should still be considered a possibility. Surgical intervention is effective in treating PHL.

\section{Additional file}

Additional file 1: Data of Patients. (XLSX $10 \mathrm{~kb}$ )

\begin{abstract}
Abbreviations
AFP: Alpha-Fetoprotein; AIDS: Acquired Immunity Deficiency Syndrome; CA125: Carbohydrate Antigens 125; CA199: Carbohydrate Antigens 199; CEA: Carcinoembryonic; CT: Computed Tomography; EBV: Epstein-Barr Virus; FNA: Fine Needle Aspiration; GIST: Gastrointestinal Stromal Tumors; IH: Immunohistochemistry; MRI: Magnetic Resonance Imaging; PHL: Primary Hepatic Leiomyoma; SMA: Smooth Muscle Actin; UA: Abdominal Ultrasound
\end{abstract}

\section{Acknowledgements}

Not applicable.

\section{Authors' contributions}

BJ designed the study and wrote this paper. ZJ and PG collected data. YL contributed to interpretation of data and reviewed the final manuscript. All authors have read and approved the final manuscript.

\section{Funding}

There was no funding source for this case report.

Availability of data and materials

All data generated or analysed during this study are included in this published article and its supplementary information files (Additional file 1).

Ethics approval and consent to participate

The study was approved by the ethical committee of the First Hospital of Jilin University.

\section{Consent for publication}

Written consent to publish was obtained from the patient for the publication of all data and accompanying images. A copy of this consent is availible for review by the editor of the journal.

Competing interests

The authors declare that they have no competing interests. 


\section{Author details}

'Department of Hepatobiliary and Pancreatic Surgery, the First Hospital of Jilin University, Changchun 130021, Jilin, China. ${ }^{2}$ Department of Breast Surgery, the First Hospital of Jilin University, Changchun 130021, Jilin, China.

\section{Received: 27 February 2019 Accepted: 30 August 2019}

\section{Published online: 07 October 2019}

\section{References}

1. Luo XZ, Ming CS, Chen XP, Gong NQ. Epstein-Barr virus negative primary hepatic leiomyoma: case report and literature review. World J Gastroenterol. 2013;19(25):4094-8.

2. Sclabas GM, Maurer CA, Wente MN, Zimmermann A, Buchler MW. Case report: hepatic leiomyoma in a renal transplant recipient. Transplant Proc. 2002:34(8):3200-2.

3. Hawkins EP, Jordan GL, McGavran MH. Primary leiomyoma of the liver. Successful treatment by lobectomy and presentation of criteria for diagnosis. Am J Surg Pathol. 1980;4(3):301-4.

4. Belli G, Ciciliano F, Lannelli A, Marano I. Hepatic resection for primary giant leiomyoma of the liver. HPB : the official journal of the International Hepato Pancreato Biliary Association. 2001;3(1):11-2.

5. Hollands MJ, Jaworski R, Wong KP, Little JM. A leiomyoma of the liver. HPB Surg : a world journal of hepatic, pancreatic and biliary surgery. 1989;1(4): 337-43.

6. Chelimilla H, Badipatla K, Ihimoyan A, Niazi M. A rare occurrence of primary hepatic leiomyosarcoma associated with epstein barr virus infection in an AIDS patient. Case Rep Gastrointest Med. 2013;2013:691862.

7. Demel R. Ein operierter Fall von Leber-Myom. Virchows Archiv. 1926;261(3): 881-4.

8. Rios-Dalenz JL. Leiomyoma of the liver. Arch Pathol. 1965;79:54-6.

9. Ishak KG, Rabin L. Benign tumors of the liver. Med Clin North Am. 1975; 59(4):995-1013.

10. Herzberg AJ, MacDonald JA, Tucker JA, Humphrey PA, Meyers WC. Primary leiomyoma of the liver. Am J Gastroenterol. 1990;85(12):1642-5.

11. Bartoli S, Alo P, Leporelli P, Puce E, Di Tondo U, Thau A. Primary hepatic leiomyoma. Minerva Chir. 1991;46(13-14):777-9.

12. Reinertson TE, Fortune JB, Peters JC, Pagnotta I, Balint JA. Primary leiomyoma of the liver. A case report and review of the literature. Dig Dis Sci. 1992;37(4):622-7.

13. Yanase $\mathrm{M}$, Ikeda $\mathrm{H}$, Ogata I, et al. Primary smooth muscle tumor of the liver encasing hepatobiliary cystadenoma without mesenchymal stroma. Am J Surg Pathol. 1999;23(7):854-9.

14. Mesenas SJ, Ng KY, Raj P, Ho JM, Ng HS. Primary leiomyoma of the liver Singap Med J. 2000:41(3):129-31.

15. Kanazawa $N$, Izumi $N$, Tsuchiya $K$, et al. A case of primary leiomyoma of the liver in a patient without evidence of immunosuppression. Hepatol Res. 2002;24(1):80.

16. Beuzen F, Roudie J, Moali I, Maitre S, Barthelemy P, Smadja C. Primary leiomyoma of the liver: a rare benign tumor. Gastroenterologie clinique et biologique. 2004;28(11):1169-72.

17. Imasato $\mathrm{M}$, Tono $\mathrm{T}$, Kano $\mathrm{T}$, et al. Primary leiomyoma of the liver: a case report. Nihon Geka Gakkai zasshi. 2005:106(11):725-9

18. Urizono $\mathrm{Y}, \mathrm{Ko} \mathrm{S}$, Kanehiro $\mathrm{H}$, et al. Primary leiomyoma of the liver: report of a case. Surg Today. 2006;36(7):629-32.

19. Marin D, Catalano C, Rossi M, et al. Gadobenate dimeglumine-enhanced magnetic resonance imaging of primary leiomyoma of the liver. J Magnetic Reson Imaging : JMRI. 2008;28(3):755-8.

20. Sousa HT, Portela F, Semedo L, et al. Primary leiomyoma of the liver: accurate preoperative diagnosis on liver biopsy. BMJ Case Rep. 2009:2009.

21. Kalil AN, Ferreira MT, Ressler F, Zettler C. Hepatic leyomioma in an immunocompetent patient. Rev Col Bras Cir. 2009;36(4):362-3.

22. Santos I, Valls C, Leiva D, Serrano T, Martinez L, Ruiz S. Primary hepatic leiomyoma: case report. Abdom Imaging. 2011;36(3):315-7.

23. Perini MV, Fink MA, Yeo DA, et al. Primary liver leiomyoma: a review of this unusual tumour. ANZ I Surg. 2013;83(4):230-3.

24. Vyas S, Psica A, Watkins J, Yu D, Davidson B. Primary hepatic leiomyoma: unusual cause of an intrahepatic mass. Ann Transl Med. 2015;3(5):73.

25. Navarro C, Hamidian Jahromi A, Donato M, Caliri N, Tempra A, Sangster G. Primary leiomyoma of the liver: case report and review of the literature. J La State Med Soc: official organ of the Louisiana State Medical Society. 2015; 167(3):129-33
26. Sadler M, Mays WL, Albert P, Javors B. Hepatic leiomyomas in two adult patients with AIDS: intravenous contrast-enhanced CT and MR imaging. Emerg Radiol. 2002;9(3):175-7.

27. Omiyale AO. Primary leiomyoma of the liver: a review of a rare tumour. HPB Surg : a world journal of hepatic, pancreatic and biliary surgery. 2014;2014: 959202.

\section{Publisher's Note}

Springer Nature remains neutral with regard to jurisdictional claims in published maps and institutional affiliations.

\section{Ready to submit your research? Choose BMC and benefit from}

- fast, convenient online submission

- thorough peer review by experienced researchers in your field

- rapid publication on acceptance

- support for research data, including large and complex data types

- gold Open Access which fosters wider collaboration and increased citations

- maximum visibility for your research: over $100 \mathrm{M}$ website views per year

At $\mathrm{BMC}$, research is always in progress.

Learn more biomedcentral.com/submissions 